\title{
Herbsttagung der Sektion Pathophysiologie und Aerosolmedizin - von der Funktionsdiagnostik zum Atemkondensat
}

H. Wuthe

Die Entwicklung der obstruktiven Lungenerkrankungen wird entscheidend von den Ursachen und dem Verlauf der Entzündungsprozesse beeinflusst. Daher nehmen in den letzten Jahren auch in unserer Sektion die Themen, die sich mit der Diagnostik und Therapie der Entzündungsprozesse beschäftigen, zu. Insbesondere die nicht invasiven Kondensat- und Exhalat-Methoden stehen im Mittelpunkt des wissenschaftlichen Interesses.

In der Atemfunktionsdiagostik, die seit Jahrzehnten erforscht wird, bemüht man sich derzeit, feststehende Regeln aus den bisherigen Erkenntnissen abzuleiten. So hat die Leitlinie der Deutschen Atemwegsliga zur Diagnostik und Therapie von Patienten mit chronisch obstruktiver Bronchitis und Lungenemphysem das Ziel, vergleichbare Prozeduren, zu ermöglichen. Diese Regeln sind für die praktische Arbeit bis hin zur Begutachtung wertvoll. Die Zusammenstellung zeigt aber auch deutlich, dass bisher nur für wenige Methoden der Funktionsdiagnostik ein international bestätigter Evidenzgrad A erreicht werden konnte. Das wissenschaftliche Interesse an Methoden, die optimal die funktionellen Störungen bei den verschiedenen Krankheiten beschreiben, bleibt daher ungemindert. Es werden weiterhin Methoden gesucht, die im besten Falle unabhängig von der aktiven Mitarbeit unter Ruheatmungsbedingungen für das breite Spektrum an Mensch und Tier, im Wach- und Schlafzustand und für alle Altersbereiche den Funktionszustand der Atmung objektiv beschreiben. Unter diesen Gesichtspunkten werden auch die Impulsoszillometrie, die Aerosol-Methoden und die Computertomographie eingesetzt und geprüft. 